\title{
Is labor-onset hypertension a novel category among hypertensive disorders of pregnancy associated with adverse events in high-risk subjects? Lights and shadows
}

\author{
Paolo Di Giosia, Paolo Giorgini and Claudio Ferri \\ Hypertension Research (2016) 39, 401-403; doi:10.1038/hr.2016.10; published online 11 February 2016
}

$\mathrm{H}$ ypertensive disorders of pregnancy are a spectrum of diseases ranging from chronic and gestational hypertension to eclampsia. Hypertensive disorders are estimated to affect $6-8 \%$ of all pregnancies worldwide. ${ }^{1,2}$ Despite being the leading cause of maternal death and a major cause of maternal and perinatal morbidity, the mechanisms responsible for the pathogenesis of hypertensive disorders of pregnancy have not yet been thoroughly elucidated.

Pregnant women are a high-risk population for hypertensive disorders because, during pregnancy, several physiological changes can lead to increased stress on the cardiovascular system. ${ }^{3}$ Within the first trimester, the total intravascular volume increases, resulting in relative dilutional anemia. An increased intravascular volume associated with neurohormonal changes leads to vasodilation. At the same time, the maternal heart rate and, in turn, cardiac output increase. ${ }^{3}$ Overall, these hemodynamic changes improve placental perfusion, which works at low pressures and high fluxes to allow fetal growth (Figure 1). However, these adjustments to the pregnancy status can have significant consequences on the maternal blood pressure control. In this context, abnormal placentation is considered to be a key issue in the promotion of hypertensive disorders of pregnancy. In fact, aberrant

P Di Giosia, P Giorgini and C Ferri are at Department of Life, Health and Environmental Sciences, University of L'Aquila, L'Aquila, Italy

E-mail: paolo.dig@hotmail.it interaction between the fetus and mother and placental hypoperfusion trigger neurohormonal feedback that promotes hypertensive maternal responses aimed at preserving placental perfusion (Figure 1). ${ }^{3}$ Pre-gravid cardiovascular diseases, such as hypertension, obesity and diabetes, facilitate and/or exacerbate the development of these adverse events. Overall, these negative hemodynamic changes can prompt hypertensive disorders of pregnancy, such as gestational hypertension, preeclampsia, eclampsia and Hemolysisis, Elevated Liver enzyme levels and Low Platelet count syndrome, as well as stroke and other cardiovascular diseases during pregnancy, delivery and the postpartum period (Figure 1).

The interesting study by Ohno et al. ${ }^{4}$ sought to clarify the perinatal outcomes and risk factors for hypertension that are first detected after the onset of labor. The study was conducted on a large population of Japanese parturient women not affected by gestational hypertensive disorders until childbirth. The authors found that nearly one-quarter of the study population developed hypertension during labor, and they identified age, body mass index, systolic blood pressure, proteinuria and severe edema as risk factors for severe/emergent labor-onset hypertension. The findings are interesting and quite innovative. Ohno et al. have first investigated, as the main outcome, the blood pressure changes during delivery. Moreover, the authors have suggested that labor-onset hypertension can be considered an independent category. In this context, there are several classifications for hypertensive disorders during pregnancy. For instance, European guidelines identify four categories: pre-existing hypertension, gestational hypertension, pre-existing hypertension plus superimposed gestational hypertension with proteinuria, and antenatally unclassifiable hypertension (Figure 2). ${ }^{5}$ Despite some differences, none of the international guidelines have proposed labor-onset hypertension as an independent category. However, we believe that the intriguing hypothesis of Ohno et al. should be tested in larger, well designed studies. Furthermore, the biological mechanisms proving this suggestion should be investigated and elucidated.

Labor is a particular physiological situation involving major hemodynamic changes that can considerably affect blood pressure. To date, few studies have explored the pathways involved in blood pressure changes at delivery. In this context, Long et al. ${ }^{6}$ suggested that the activation of renin-angiotensin system at delivery is associated with higher blood pressure. At the same time, it is known that blood pressure rises as a result of the effect of uterine contractions, causing an increase in both stroke volume and heart rate. ${ }^{7}$ In addition, acute pain during labor may lead to a generalized increase in sympathetic nerve activity, which is responsible for a higher cardiac output and the elevation of blood pressure. $^{8}$

Moreover, Ohno et al. have concluded that late-onset hypertension is a risk factor for emergent hypertension and is associated with 


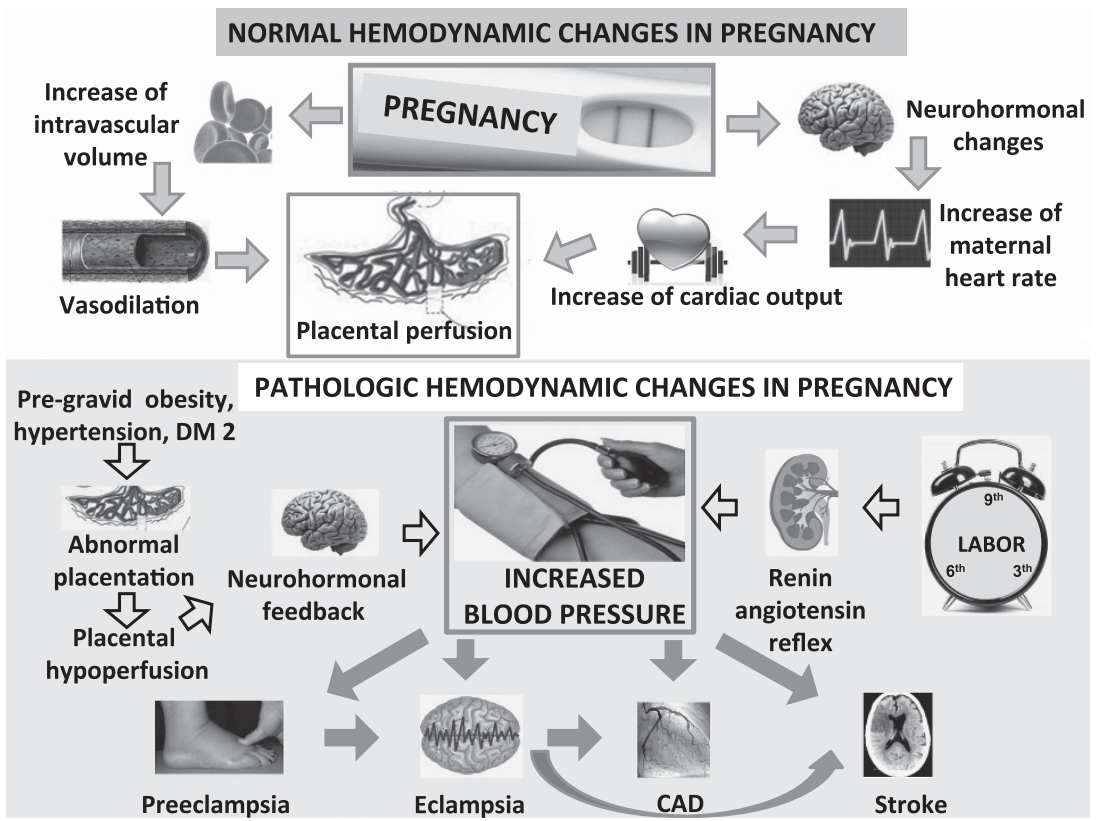

Figure 1 Physiological (above) and pathological (below) hemodynamic changes during pregnancy. CAD, coronary artery disease; DM 2, diabetes mellitus type 2.

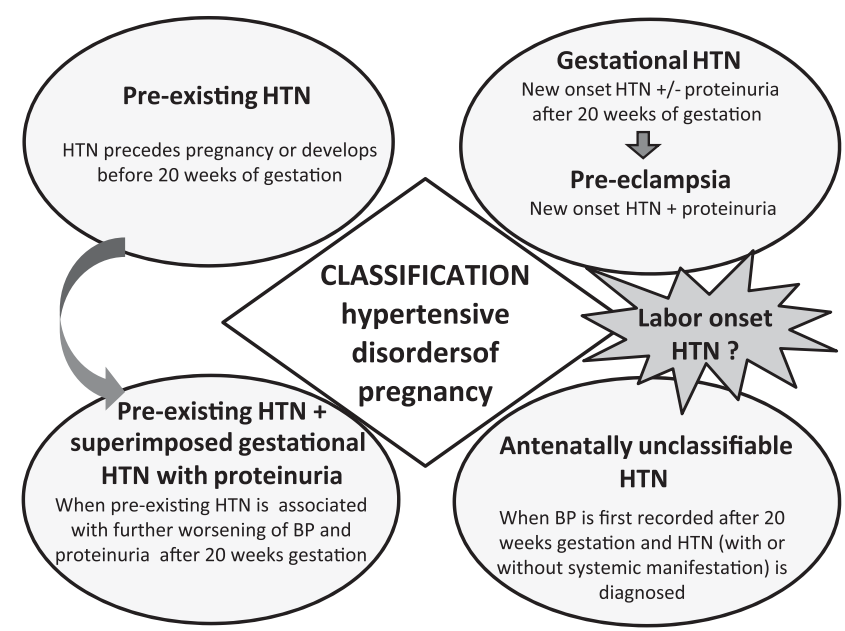

Figure 2 Classification of hypertensive disorders during pregnancy by the European Society of Cardiology. Is there a role for labor-onset hypertension? BP, blood pressure; HTN, hypertension $(B P>140 / 90)$; Proteinuria means $\geqslant 0.3 \mathrm{~g}$ per day in a 24 - $h$ urine collection or $\geqslant 30 \mathrm{mg} \mathrm{mmol}^{-1}$ urinary creatinine in a spot random urine sample.

eclampsia and stroke during the perinatal period. Similarly, high blood pressure levels have recently been associated with an increase in early postpartum preeclampsia. In a 6month prospective observational study that included 1435 women, increased blood pressure values predicted the onset of early de novo postpartum preeclampsia in parturient women without any hypertensive disease during or before pregnancy. ${ }^{9}$ Recent studies have proposed anthropometric measurements (that is, body mass index and waist-to-hip ratio) $)^{10}$ and parameters of vascular function (central systolic pressure, augmentation index and pulse wave velocity) $)^{11,12}$ as valid predictors of preeclampsia.

In the study by Ohno et al., ${ }^{4}$ there are some limitations that merit mentioning. First, although it has been reported that eclampsia episodes and pregnancy-associated strokes often occur during labor and are associated with elevated blood pressure at delivery in the absence of any prior symptoms of hypertension or proteinuria, ${ }^{13}$ these associations are not sufficient to support that late-onset hypertension is a risk factor for eclampsia and stroke. Second, the size and homogeneity of the sample limit the generalizability of this study. Finally, the study does not consider the administration of certain agents such as epidural analgesia and oxytocin, which may influence blood pressure during delivery. In particular, it has been reported that oxytocin moderately increases systolic blood pressure during labor. ${ }^{14}$ However, several studies have established that epidural block may cause a drop in the blood pressure values and may have a role in reducing the risk of late-onset hypertension. ${ }^{15}$ In keeping with these findings, it would be fascinating to explore the impact of these agents on blood pressure values and outcomes. In addition, on the basis of previous studies, the authors have suggested that the correct management of blood pressure during labor could reduce maternal deaths and strokes. Although few retrospective studies support this hypothesis, further investigations are required to identify the link between labor-onset hypertension and the risk of stroke to design an adequate treatment strategy.

In conclusion, although the clinical management of hypertension in pregnancy is well established, ${ }^{16}$ Ohno et al. have suggested a novel category of hypertension disorder, resulting in adverse pregnancy outcomes. As a result, their findings urge clinicians to promote the accurate measurement and prompt management of blood pressure during labor, especially in patients with risk factors for cardiovascular diseases, to reduce maternal and perinatal mortality and morbidity.

\section{CONFLICT OF INTEREST}

The authors declare no conflict of interest.

1 Jim B, Sharma S, Kebede T, Acharya A. Hypertension in pregnancy: a comprehensive update. Cardiol Rev 2010; 18: 178-189.

2 Zareian Z. Hypertensive disorders of pregnancy. Int J Gynaecol Obstet 2004; 87: 194-198.

3 Yoder SR, Thornburg LL, Bisognano JD. Hypertension in pregnancy and women of childbearing age. Am J Med 2009; 122: 890-895.

4 Ohno Y, Terauchi M, Tamakoshi K, Shiozaki A, Saito $S$. The risk factors for labor onset hypertension. Hypertens Res 2016; 39: 260-265.

5 ESC Committee for Practice Guidelines. ESC Guidelines on the management of cardiovascular diseases during pregnancy: the Task Force on the Management of Cardiovascular Diseases during Pregnancy of the European Society of Cardiology (ESC). Eur Heart J 2011; 32: 3147-3197.

6 Long PA, Oats JN, Beischer NA. Labour onset preeclampsia. Aust N Z J Obstet Gynaecol 1981; 21: 16-19.

7 Robson SC, Dunlop W, Boys RJ, Hunter S. Cardiac output during labour. Br Med J (Clin Res Ed) 1987; 295: 1169-1172.

8 Ghione S, Rosa C, Mezzasalma L, Panattoni E. Arterial hypertension is associated with hypalgesia in humans. Hypertension 1988; 12: 491-497. 
9 Cohen J, Vaiman D, Sibai BM, Haddad B. Blood pressure changes during the first stage of labor and for the prediction of early postpartum preeclampsia: a prospective study. Eur J Obstet Gynecol Reprod Biol 2015; 184: 103-107.

10 Taebi M, Sadat Z, Saberi F, Kalahroudi MA. Early pregnancy waist-to-hip ratio and risk of preeclampsia: a prospective cohort study. Hypertens Res 2015; 38: 80-83.

11 Katsipi I, Stylianou K, Petrakis I, Passam A, Vardaki E, Parthenakis F, Makrygiannaki A, Daphnis E, Kyriazis J. The use of pulse wave velocity in predicting pre-eclampsia in high-risk women. Hypertens Res 2014; 37: 733-740.

12 Tomimatsu T, Fujime M, Kanayama T, Mimura K, Koyama S, Kanagawa T, Endo M, Shimoy K, Kimura T. Abnormal pressure-wave reflection in pregnant women with chronic hypertension: association with maternal and fetal outcomes. Hypertens Res 2014; 37: 989-992.

13 Ohno Y, Furuhashi M, Ishikawa K, Kaseki S, Kondo H, Kikkawa F. Results of a questionnaire survey on pregnancy-associated stroke from 2005 to 2012 in Aichi Prefecture, Japan. Hypertens Res Preg 2014; 2: 16-20.
14 Watanabe K, Suzuki Y, Yamamoto T. Incidence of eclampsia in Japanese women. Hypertens Res Preg 2013; 1: 31-34.

15 Pinder AJ, Dresner M, Calow C, Shorten GD, O'Riordan J, Johnson R. Haemodynamic changes caused by oxytocin during caesarean section under spinal anaesthesia. Int J Obstet Anesth 2002; 11: 156-159.

16 Borghi C, Ferri C, Sechi L, Italian Society of Hypertension. Clinical management of hypertension in pregnancy. Practical recommendations from the Italian Society of Hypertension (SIIA). High Blood Press Cardiovasc Prev 2013; 20: 123-127. 